\title{
Locating Multiple Facilities in Convex Sets with Fuzzy Data and Block Norms
}

\author{
Jafar Fathali, Ali Jamalian \\ Department of Mathematics, Shahrood University of Technology, Shahrood, Iran \\ Email: fathali@shahroodut.ac.ir, a.jamalian.math@gmail.com
}

Received July 18, 2012; revised August 18, 2012; accepted August 25, 2012

\begin{abstract}
In this paper we study the problem of locating multiple facilities in convex sets with fuzzy parameters. This problem asks to find the location of new facilities in the given convex sets such that the sum of weighted distances between new facilities and existing facilities is minimized. We present a linear programming model for this problem with block norms, then we use it for problems with fuzzy data. We also do this for rectilinear and infinity norms as special cases of block norms.
\end{abstract}

Keywords: Multifacility Location; Block Norm; Minisum; Fuzzy Data; Linear Programming

\section{Introduction}

Within the wide interaction between operations research (OR) and computer science, a major application area concerns locational decisions. The locational decisions determine how to use the best case possible for achievement of activities with respect to existence a tools in our purpose direction. This aim is mostly contained of locating facilities. Multiple facility location problem is a well-known problem in operations research and especially in locational analysis and has been studied in depth, for more details see [1,2]. Locating facilities with some constraints on the regions which are contained facilities or customers is an interesting problem and has been studied by different constraints and conditions. Sarkar et al. [3] addressed the finite size 1-center placement problem on a rectangular plane in the presence of barriers. Barriers are regions in which both facility location and travel through are prohibited. The feasible region for facility placement is subdivided into cells along the lines by Larson and Sadiq [4]. Muoz-Pérez et al. [5] developed the problem of locating an undesirable facility in a bounded polygonal region (with forbidden polygonal zones), using Euclidean distances, under an objective function that generalizes the maximin and maxisum criteria, and includes other criteria such as the linear combinations of these criterions. Bhattacharya et al. [6], presented a fuzzy goal programming model for locating a single facility on a plane bounded by a convex polygon under the triple criteria maximin, minimax and minisum location. In [7] presented a fuzzy goal programming model for locating multiple new facilities on a plane bounded by a convex polygon under the criteria: 1) minimize the sum of all the transportation costs and 2) minimize the maximum distances from the facilities to the demand points. It has also been proved that the given methodology, always gives a nondominated solution. Nayeem and Pal [8] consider a facility location problem called p-center on fuzzy networks.

In this paper we present a model for locating multiple new facilities in convex sets with respect to multiple existing facilities and demand points, then present a linear programming model for this problem with block norms. We use this results for the problem with fuzzy data. We also do this for rectilinear and infinity norms as special cases of block norms. Rectilinear distances have been taken as the scenario may be thought of in an urban setting. Study of this problem and its modeling has many applications in industry such as locating machines in a workshop.

Let $m$ existing facilities be located at the known distinct points $P_{1}, \cdots, P_{m}$ in the plane. In a multifacility location problem the optimal location of $n$ new facilities $X_{1}, \cdots, X_{n}$ is sought with respect to the set of existing facilities. Let $d\left(X_{j}, P_{i}\right)$ represents the distance between the locations of new facility $j$ and existing facility $i$, and $d\left(X_{j}, X_{k}\right)$ be the distance between the locations of new facilities $j$ and $k$.

Let the cost per unit distance between new facility $j$ and existing facility $i$ be denoted by $w_{j i}$ and $v_{j k}$ being the corresponding cost per unit distance between new facilities $j$ and $k$. The total transportation cost associated with new facilities located at $X_{1}, \cdots, X_{n}$ is 
given by

$$
\begin{aligned}
f\left(X_{1}, \cdots, X_{n}\right) & =\sum_{1 \leq j<k \leq n} v_{j k} d\left(X_{j}, X_{k}\right) \\
& +\sum_{j=1}^{n} \sum_{i=1}^{m} w_{j i} d\left(X_{j}, P_{i}\right) .
\end{aligned}
$$

The multifacility location problem can be stated as the selection of locations $X_{1}^{*}, \cdots, X_{n}^{*}$ of new facilities such that total cost is minimized. For more details in location theory see [4,9-12].

The block norms are norms which their contours are polytopes. For example $l_{1}$ and $l_{\infty}$ are two block norms. The first application of block norms to solve the location problems suggested by Ward and Wendell $[13,14]$. They are shown that a block norm can be characterized as follows:

$$
\|x\|_{B}=\min \left\{\sum_{g=1}^{r}\left|\lambda_{g}\right|: x=\sum_{g=1}^{r} \lambda_{g} b_{g}\right\}
$$

where the points $b_{g}$ and $-b_{g}$ with $g=1, \cdots, r$ form the extreme points of the polytope corresponding to the unit contour. They also presented another characterization based on polar set for block norms. This characterization follows:

$$
\|x\|_{B}=\max \left\{\left|x b_{g}^{0}\right|: g=1, \cdots, r^{0}\right\}
$$

where $b_{g}^{0}$ and $-b_{g}^{0}$ with $g=1, \cdots, r^{0}$ are extreme points of the polar set

$$
B^{0}=\left\{v: b_{g} v \leq 1 \text { for all } g= \pm 1, \pm 2, \cdots, \pm r\right\} .
$$

By using these characterizations Ward and Wendell $[13,14]$ shown that the minimax and minisum single facility location problems can be written as a LP problems.

If a block norm $B$, is applied to measure the distances in the plane then we can write a linear programming for the problem in two ways:

1) For $i=1, \cdots, n$ let

$$
\left\|X-P_{i}\right\|_{B}=\sum_{g=1}^{r}\left(\lambda_{g i}^{+}+\lambda_{g i}^{-}\right), X-P_{i}=\sum_{g=1}^{r}\left(\lambda_{g i}^{+}-\lambda_{g i}^{-}\right) b_{g} .
$$

2) For $i=1, \cdots, n$ let

$$
z_{i}=\left\|X-P_{i}\right\|_{B}=\max \left\{\left|\left(X-P_{i}\right) b_{g}^{0}\right|: g=1, \cdots, r^{0}\right\} .
$$

By substituting these in models we have a linear programming. In this paper we assume that distance is measured by block norm and as a special case rectilinear norm in two dimensional space. When $X_{j}=\left(x_{j}, y_{j}\right)$ and $P_{i}=\left(p x_{i}, p y_{i}\right)$, then objective function is as below:

$$
\begin{aligned}
f\left(X_{1}, \cdots, X_{n}\right) & =\sum_{1 \leq j<k \leq n} v_{j k}\left\|X_{j}-X_{k}\right\|_{B} \\
& +\sum_{j=1}^{n} \sum_{i=1}^{m} w_{j i}\left\|X_{j}-P_{i}\right\|_{B} .
\end{aligned}
$$

If we use the rectilinear norm for measuring distances between points we have:

$$
\begin{aligned}
& d\left(X_{j}, X_{k}\right)=\left|x_{j}-x_{k}\right|+\left|y_{j}-y_{k}\right| \\
& d\left(X_{j}, P_{i}\right)=\left|x_{j}-p x_{i}\right|+\left|y_{j}-p y_{i}\right| .
\end{aligned}
$$

On substituting (5) and (6) into (1) and rearranging terms, we obtain

$$
f\left(X_{1}, \cdots, X_{n}\right)=f_{1}\left(x_{1}, \cdots, x_{n}\right)+f_{2}\left(y_{1}, \cdots, y_{n}\right)
$$

where

$$
\begin{aligned}
f_{1}\left(x_{1}, \cdots, x_{n}\right) & =\sum_{1 \leq j<k \leq n} v_{j k} d\left(x_{j}, x_{k}\right) \\
& +\sum_{j=1}^{n} \sum_{i=1}^{m} w_{j i} d\left(x_{j}, p x_{i}\right)
\end{aligned}
$$

And

$$
\begin{aligned}
f_{2}\left(y_{1}, \cdots, y_{n}\right) & =\sum_{1 \leq j<k \leq n} v_{j k} d\left(y_{j}, y_{k}\right) \\
& +\sum_{j=1}^{n} \sum_{i=1}^{m} w_{j i} d\left(y_{j}, p y_{i}\right)
\end{aligned}
$$

The expressions $f_{1}$ and $f_{2}$ give the total cost incurred due to "travel" in the $x$ and $y$ directions, respectively.

From (7), it follows that

$$
\begin{aligned}
\min f\left(X_{1}, \cdots, X_{n}\right) & =\min f_{1}\left(x_{1}, \cdots, x_{n}\right) \\
& +\min f_{2}\left(y_{1}, \cdots, y_{n}\right) .
\end{aligned}
$$

We minimize $f_{1}$ with constraints that new facilities be in convex sets, by transforming it to an equivalent LP problem will provide optimum $x$ coordinate of the new facilities. An exactly analogous procedure is used to minimize $f_{2}$.

In Section 2 we introduce a model for locating multiple facilities in convex sets. In Section 3 we give a practical exampl. A method for fuzzy linear programming problems and locating for fuzzy data are introduced in Section 4. Conclusion is achieved in the last section.

\section{Locating Multiple Facilities in Convex Sets}

Suppose that we have a set of $m$ machines in a workshop for which their captured spaces are intervals. Assume that $\left(p x_{j}, p y_{j}\right), j=1, \cdots, m$ corresponding to the coordinates of machines. Also, suppose that we divide the remaining region of the workshop to $K$ convex regions (here we consider convex regions as rectangle ones) which are given as follows.

$$
S_{j}=\left\{(x, y) \mid a_{j} \leq x \leq b_{j}, c_{j} \leq y \leq d_{j}\right\} \text { for } j=1, \cdots, K .
$$

Now, we want to find $n$ points in these $K$ regions 
to locate new machines $X_{1}=\left(x_{1}, y_{1}\right), \cdots, X_{n}=\left(x_{n}, y_{n}\right)$ such that the sum of movement costs between each two new facilities and also new facilities and machines is minimized. We assume the movement cost between new facilities and machines to be proportional to the distance between them with a weight. Let $v_{j k}$ be the weight of movement between facilities $j$ and $k$, and $w_{j i}$ be the weight of movement between facility $j$ and machine $k$. Then we have the following problem.

$$
\begin{aligned}
& \min Z=\sum_{1 \leq j<k \leq n} v_{j k} d\left(x_{j}, x_{k}\right)+\sum_{j=1}^{n} \sum_{i=1}^{m} w_{j i} d\left(x_{j}, p x_{i}\right) \\
& +\sum_{1 \leq j<k \leq n} v_{j k} d\left(y_{j}, y_{k}\right)+\sum_{j=1}^{n} \sum_{i=1}^{m} w_{j i} d\left(y_{j}, p y_{i}\right) \\
& \text { S.t. } a_{i} \leq x_{j} \leq b_{i}, i=1, \cdots, K, j=1, \cdots, n, \\
& c_{i} \leq y_{j} \leq d_{i}, i=1, \cdots, K, j=1, \cdots, n .
\end{aligned}
$$

In this paper we consider the special case that only one machine is assigned to each region and for each machine, only one region contains it. In fact, this part of problem reduces to an assignment problem. So we define binary variables $\gamma_{i j}$ which are equal to 1 if machine $j$ is assigned to region $i$ and are equal to 0 otherwise, and replace the constraints of the problem with the following.

$$
\begin{aligned}
& x_{j} \leq b_{i}+M\left(1-\gamma_{i j}\right), i=1, \cdots, K, j=1, \cdots, n \\
& x_{j} \geq a_{i}-M\left(1-\gamma_{i j}\right), i=1, \cdots, K, j=1, \cdots, n \\
& y_{j} \leq d_{i}+M\left(1-\gamma_{i j}\right), i=1, \cdots, K, j=1, \cdots, n \\
& y_{j} \geq c_{i}-M\left(1-\gamma_{i j}\right), i=1, \cdots, K, j=1, \cdots, n \\
& \sum_{i=1}^{K} \gamma_{i j}=1, j=1, \cdots, n ; \sum_{j=1}^{n} \gamma_{i j} \leq 1, i=1, \cdots, K, \gamma_{i j} \in\{0,1\}
\end{aligned}
$$

where $M$ is an upper bound for variables. This constraints ensure that only one machine is assigned to each region and for each machine, only one region contains it.

\subsection{The Problem with Block Norms}

Suppose the distances are measured by a block norm $B$, then we can write the problem as follows.

$$
\begin{gathered}
\min Z=\sum_{1 \leq j<k \leq n} v_{j k}\left\|X_{j}-X_{k}\right\|_{B}+\sum_{j=1}^{n} \sum_{i=1}^{m} w_{j i}\left\|X_{j}-P_{i}\right\|_{B} \\
\text { S.t. } x_{j} \leq b_{i}+M\left(1-\gamma_{i j}\right), i=1, \cdots, K, j=1, \cdots, n \\
x_{j} \geq a_{i}-M\left(1-\gamma_{i j}\right), i=1, \cdots, K, j=1, \cdots, n \\
y_{j} \leq d_{i}+M\left(1-\gamma_{i j}\right), i=1, \cdots, K, j=1, \cdots, n \\
y_{j} \geq c_{i}-M\left(1-\gamma_{i j}\right), i=1, \cdots, K, j=1, \cdots, n \\
\sum_{i=1}^{K} \gamma_{i j}=1, j=1, \cdots, n ; \sum_{j=1}^{n} \gamma_{i j} \leq 1, i=1, \cdots, K, \gamma_{i j} \in\{0,1\} .
\end{gathered}
$$

So by definition of block norms and using transformations for the problem by variables $\lambda_{g j k}^{+}, \lambda_{g j k}^{-}$, we have

$$
\begin{aligned}
& \min Z=\sum_{1 \leq j<k \leq n g=1} \sum_{j k}^{r} v_{g j}\left(\lambda_{g j k}^{+}+\lambda_{g j k}^{-}\right) \\
& \quad+\sum_{j=1}^{n} \sum_{i=1}^{m} \sum_{g=1}^{r} w_{j i}\left(\beta_{g j i}^{+}+\beta_{g j i}^{-}\right) \\
& \text {S.t. } X_{j}-X_{k}=\sum_{g=1}^{r}\left(\lambda_{g j k}^{+}-\lambda_{g j k}^{-}\right) b_{g}, \\
& X_{j}-P_{i}=\sum_{g=1}^{r}\left(\beta_{g j i}^{+}-\beta_{g j i}^{-}\right) b_{g} \\
& x_{j} \leq b_{i}+M\left(1-\gamma_{i j}\right), i=1, \cdots, K, j=1, \cdots, n \\
& x_{j} \geq a_{i}-M\left(1-\gamma_{i j}\right), i=1, \cdots, K, j=1, \cdots, n \\
& y_{j} \leq d_{i}+M\left(1-\gamma_{i j}\right), i=1, \cdots, K, j=1, \cdots, n \\
& y_{j} \geq c_{i}-M\left(1-\gamma_{i j}\right), i=1, \cdots, K, j=1, \cdots, n \\
& \sum_{i=1}^{K} \gamma_{i j}=1, j=1, \cdots, n ; \sum_{j=1}^{n} \gamma_{i j} \leq 1, i=1, \cdots, K, \\
& \gamma_{i j} \in\{0,1\}, \lambda_{g j k}^{+}, \lambda_{g j k}^{-}, \beta_{g j i}^{+}, \beta_{g j i}^{-} \geq 0
\end{aligned}
$$

which is a linear programming model.

\subsection{The Problem with Rectilinear Norm}

With rectilinear norm, model (10) is convertible to the following model:

$$
\begin{gathered}
\min Z=\sum_{1 \leq j<k \leq n} v_{j k}\left|x_{j}-x_{k}\right|+\sum_{j=1}^{n} \sum_{i=1}^{m} w_{j i}\left|x_{j}-p x_{i}\right| \\
+\sum_{1 \leq j<k \leq n} v_{j k}\left|y_{j}-y_{k}\right|+\sum_{j=1}^{n} \sum_{i=1}^{m} w_{j i}\left|y_{j}-p y_{i}\right| \\
\text { S.t. } x_{j} \leq b_{i}+M\left(1-\gamma_{i j}\right), i=1, \cdots, K, j=1, \cdots, n \\
x_{j} \geq a_{i}-M\left(1-\gamma_{i j}\right), i=1, \cdots, K, j=1, \cdots, n \\
y_{j} \leq d_{i}+M\left(1-\gamma_{i j}\right), i=1, \cdots, K, j=1, \cdots, n \\
y_{j} \geq c_{i}-M\left(1-\gamma_{i j}\right), i=1, \cdots, K, j=1, \cdots, n \\
\sum_{i=1}^{K} \gamma_{i j}=1, j=1, \cdots, n ; \sum_{j=1}^{n} \gamma_{i j} \leq 1, i=1, \cdots, K, \gamma_{i j} \in\{0,1\} .
\end{gathered}
$$

Note that the objective function of model (13) is nonlinear. However we can linearize it as follows. Let

$$
\begin{aligned}
& x_{j}-x_{k}=p_{j k}-q_{j k}, \quad y_{j}-y_{k}=p_{j k}^{\prime}-q_{j k}^{\prime}, \\
& x_{j}-p x_{i}=r_{j i}-s_{j i} \text { and } y_{j}-p y_{k}=r_{j i}^{\prime}-s_{j i}^{\prime}
\end{aligned}
$$

for $i=1, \cdots, K, j=1, \cdots, n, k=1, \cdots, m$.

If

$$
p_{j k} q_{j k}=0, p_{j k} \geq 0, q_{j k} \geq 0
$$

then $\left|x_{j}-x_{k}\right|=p_{j k}+q_{j k}$. So by using this transformation 
in model (13), we have:

$$
\begin{aligned}
& \min Z=\sum_{1 \leq j<k \leq n} v_{j k}\left(p_{j k}+q_{j k}\right)+\sum_{j=1}^{n} \sum_{i=1}^{m} w_{j i}\left(r_{j i}+s_{j i}\right) \\
& +\sum_{1 \leq j<k \leq n} v_{j k}\left(p_{j k}^{\prime}+q_{j k}^{\prime}\right)+\sum_{j=1}^{n} \sum_{i=1}^{m} w_{j i}\left(r_{j i}^{\prime}+s_{j i}^{\prime}\right) \\
& \text { S.t. } x_{j}-x_{k}-p_{j k}+q_{j k}=0, y_{j}-y_{k}-p_{j k}^{\prime}+q_{j k}^{\prime}=0, \\
& x_{j}-r_{j i}+s_{j i}=p x_{i}, y_{j}-r_{j i}^{\prime}+s_{j i}^{\prime}=p y_{i} \\
& x_{j} \leq b_{i}+M\left(1-\gamma_{i j}\right), i=1, \cdots, K, j=1, \cdots, n \\
& x_{j} \geq a_{i}-M\left(1-\gamma_{i j}\right), i=1, \cdots, K, j=1, \cdots, n \\
& y_{j} \leq d_{i}+M\left(1-\gamma_{i j}\right), i=1, \cdots, K, j=1, \cdots, n \\
& y_{j} \geq c_{i}-M\left(1-\gamma_{i j}\right), i=1, \cdots, K, j=1, \cdots, n \\
& \sum_{i=1}^{K} \gamma_{i j}=1, j=1, \cdots, n ; \sum_{j=1}^{n} \gamma_{i j} \leq 1, i=1, \cdots, K, \\
& \gamma_{i j} \in\{0,1\}, p_{j k}, q_{j k}, p_{j k}^{\prime}, q_{j k}^{\prime}, r_{j i}, s_{j i}, r_{j i}^{\prime}, s_{j i}^{\prime} \geq 0 .
\end{aligned}
$$

Note that we have deleted the sets of multiplicative constraints, $\quad p_{j k} q_{j k}=0, \quad p_{j k}^{\prime} q_{j k}^{\prime}=0, \quad r_{j k} s_{j k}=0 \quad$ and $r_{j k}^{\prime} s_{j k}^{\prime}=0$. Since in solving model (14) by linear programming the theory of linear programming guarantees that some basic feasible solution, will be a minimum feasible solution. For any basic feasible solution, if $p_{j k}$ is in the basic feasible solution, $q_{j k}$ will not be and vice versa. Since variables not in the basic feasible solution, are zero, the multiplicative constraints will therefore be satisfied for every basic feasible solution. To see why both $p_{j k}$ and $q_{j k}$ would not be in the same basic feasible solution, suppose that the first two sets of equality constraints of model (14) are written in matrix form; then the column of the matrix corresponding to $p_{j k}$ is -1 times the column corresponding to $q_{j k}$, so that the two columns are linearly dependent. Likewise, the two columns corresponding to $r_{j i}$ and $s_{j i}$ are linearly dependent but a basis consists of linearly independent vectors. If both $p_{j k}$ and $q_{j k}$ were in the same basic feasible solution, the corresponding columns making up the basis would be linearly dependent which can not be.

\section{Examples}

In this section we give some examples for the mentioned models. We solve them by LINGO software.

Example 1. Consider the problem of locating a new machine in an existing layout consisting of five machines $P_{1}, \cdots, P_{5}$. The coordinate of machines are presented in the Table 1. Also consider the four possible regions $S_{1}, \cdots, S_{4}$, in order to locate two new machines in Table 2. The problem is to obtain an optimal location for the new machines in the region $S_{j}, j=1,2,3,4$, so that the sum of the rectilinear distances of the new machines from the other machines is minimized.
Using model (14) we obtain $X_{1}^{*}=(4,5) \in S_{3}$, $X_{2}^{*}=(3,4) \in S_{2}$ and the optimal value of objective function is $Z^{*}=30$.

Example 2. Consider six important industrial regions in a city which receive compulsory services from two fire stations. These industrial regions are shown in Table 3. Also three sites are considered for building fire stations according to Table 4. The problem is to determine two sites considering of three sites for building two fire stations so that the sum of the distances of the fire stations from the six industrial regions is minimized. Let the distances are measured by a block norm which its extreme points are

$$
\begin{aligned}
& b_{1}=(0,1), b_{2}=(\sqrt{3} / 2,1 / 2), b_{3}=(1,0), \\
& b_{4}=(\sqrt{3} / 2,-1 / 2), \\
& b_{-1}=(0,-1), b_{-2}=(-\sqrt{3} / 2,-1 / 2), \\
& b_{-3}=(-1,0) \text { and } b_{-4}=(-\sqrt{3} / 2,1 / 2) .
\end{aligned}
$$

Using model (12) we obtain the optimal solution $\left(x_{1}^{*}, y_{1}^{*}\right)=(12,18) \in S_{2}, \quad\left(x_{2}^{*}, y_{2}^{*}\right)=(32,18) \in S_{3}$ and

Table 1. The coordinate of machines.

\begin{tabular}{llllll}
\hline & $P_{1}$ & $P_{2}$ & $P_{3}$ & $P_{4}$ & $P_{5}$ \\
\hline$x$ & 4 & 2 & 2 & 4 & 6 \\
$y$ & 4 & 2 & 5 & 1 & 5 \\
\hline
\end{tabular}

Table 2. The interval coordinates of regions.

\begin{tabular}{ccccc}
\hline & $x^{L}$ & $x^{U}$ & $y^{L}$ & $y^{U}$ \\
\hline$S_{1}$ & 1 & 2 & 6 & 7 \\
$S_{2}$ & 2 & 3 & 3 & 4 \\
$S_{3}$ & 4 & 5 & 5 & 7 \\
$S_{4}$ & 5 & 6 & 2 & 3 \\
\hline
\end{tabular}

Table 3. The coordinates of industrial regions.

\begin{tabular}{ccccccc}
\hline & A & B & C & D & E & F \\
\hline$x$ & 20 & 25 & 13 & 25 & 4 & 18 \\
$y$ & 15 & 25 & 32 & 14 & 21 & 8 \\
\hline
\end{tabular}

Table 4. The interval coordinates of sites.

\begin{tabular}{lcccc}
\hline & $x^{L}$ & $x^{U}$ & $y^{L}$ & $y^{U}$ \\
\hline$S_{1}$ & 4 & 6 & 8 & 10 \\
$S_{2}$ & 10 & 12 & 18 & 23 \\
$S_{3}$ & 32 & 33 & 18 & 20 \\
\hline
\end{tabular}


$Z^{*}=138.3539$. The weights of regions and sites are the same.

\section{Fuzzy Linear Programming}

The concept of decision making in fuzzy environment was first proposed by Bellman and Zadeh [1]. Subsequently, Tanaka et al. [15] made use of this concept in mathematical programming (see also [16]). A Fuzzy Linear Programming (FLP) is concerned with the optimization (minimization or maximization) of a fuzzy linear function while satisfying a set of linear equality and/or inequality fuzzy constraints. Fuzzy linear programming problem with fuzzy coefficients was proposed by $\mathrm{Ne}-$ goita [17]. A formulation of fuzzy linear programming with fuzzy constraints and a solution method were given by Tanaka and Asai [18]. Maleki et al. [19] introduced a linear programming problem with fuzzy variables and proposed a method for solving it. Fang and $\mathrm{Hu}$ [20] consider linear programming with fuzzy constraint coefficients (see also [21]). Gasimov and Yenilmez [22] discuss solution of fuzzy linear programming problems using linear membership functions (see also [23]). Maleki [24] used a certain ranking function to solve fuzzy linear programming problems. He also introduced a new method for solving linear programming problems with vagueness in constraints using a linear ranking function. Mishmast et al. [25] introduced the lexicographic ranking function to order fuzzy numbers and solved fuzzy number linear programming problems by lexicographic ranking function. In this paper FLP with Right Hand Solution (R.H.S) is considered.

An ordered pair of functions

$$
T(\underline{U}(r), \bar{U}(r)), 0 \leq r \leq 1,
$$

is called a fuzzy number if and only if it satisfied in the following requirements.

1) $\underline{U}(r)$ is a bounded left continues non decreasing function over $[0,1]$.

2) $\bar{U}(r)$ is a bounded left continues non increasing function over $[0,1]$.

3) $\underline{U}(r)$ and $\bar{U}(r)$ are right continues in 0 .

4) $\underline{U}(r) \leq \bar{U}(r), 0 \leq r \leq 1$ where

$$
\underline{U}(r)=w r+(c-w)
$$

and

$$
\bar{U}(r)=-w r+(c+w), 0 \leq r \leq 1,
$$

which $c, w \in R, c=\operatorname{Core}(T)$ and $w=W(T) \geq 0$, $T=(c, w)$ is called Symmetric Triangular Fuzzy Number (STFN). Let ST be the set of all STFN.

A crisp number is simply represented by

$$
\underline{U}(r)=\bar{U}(r)=\alpha, 0 \leq r \leq 1 .
$$

The proofs of all the theorems in this section are given in [1].

\subsection{Theorem}

If $T=\left(c_{1}, w_{1}\right), U=\left(c_{2}, w_{2}\right)$ be STFNs, $k \in R, \tilde{X} \in S T$ and $A$ be a matrix then,

1) $T=U$ if and only if $c_{1}=c_{2}$ and $w_{1}=w_{2}$

2) $T+U=\left(c_{1}+c_{2}, w_{1}+w_{2}\right)$

3) $k T=\left(k c,|k| w_{1}\right)$

4) $A \tilde{X}=(A \operatorname{Core}(\tilde{X}) ;|A| W(\tilde{X}))$, which $|A|_{i j}=\left|a_{i j}\right|$.

\subsection{Definition}

Let $T=\left(c_{1}, w_{1}\right), U=\left(c_{2}, w_{2}\right)$ be STFNs, we say $T \tilde{<} U$ if and only if

1) $c_{1}<c_{2}$ or

2) $c_{1}=c_{2}$ and $w_{1}<w_{2}$

and $T \tilde{\leq} U$ if and only if $T \tilde{<} U$ or $T=U$.

Now consider fuzzy LP as follows

$$
\min C \tilde{X} \text { S.t. } A \tilde{X}=\tilde{b}, \tilde{X} \in S T, \tilde{X} \tilde{\geq} 0
$$

which $A \in R^{m \times n}, c \in R^{n}$ and $\tilde{b}$ is an triangular fuzzy vector. We reduce problem (15), to two following problems.

$$
\min C X \text { S.t. } A X=\operatorname{Core}(b), X \geq 0
$$

and

$$
\min |C| Y \text { S.t. }|A| Y=W(b), Y \geq 0
$$

where $|A|_{i j}=\left|a_{i j}\right|,|C|_{i}=\left|c_{i}\right|$.

\subsection{Theorem}

$\tilde{X}$ is a feasible solution problem (15) if and only if $X=\operatorname{Core}(\tilde{X})$ is a feasible solution of problem (16) and $Y=W(\tilde{X})$ is feasible solution of problem (17).

\subsection{Theorem}

$\tilde{X}^{*}$ is an optimal solution of problem (15) if and only if $X^{*}=\operatorname{Core}\left(\tilde{X}^{*}\right)$ is an optimal solution of problem (16) and $Y^{*}=W\left(\tilde{X}^{*}\right)$ is an optimal solution of problem (17).

\subsection{Locating Multiple Facilities in Convex Sets with Fuzzy Data}

Suppose that we have a set of $m$ machines in a workshop for which the captured spaces are as intervals. Assume that $\left(p \ddot{x}_{j}, p \ddot{y}_{j}\right), j=1, \cdots, m$ corresponding to their coordinates, (the sign is used for fuzzy numbers). Also, suppose that we divide the remaining region of the workshop to $K$ convex regions (here we consider convex regions as rectangle ones) which are given as fol- 
lows:

$$
S_{j}=\left\{(x, y) \mid \tilde{a}_{j} \tilde{\leq} x \tilde{\leq} \tilde{b}_{j}, \tilde{c}_{j} \tilde{\leq} y \tilde{\leq} \tilde{d}_{j}\right\} \text { for } j=1, \cdots, K
$$

where $\tilde{a}_{j}, \tilde{b}_{j}, \tilde{c}_{j}$ and $\tilde{d}_{j}$ for $j=1, \cdots, K$ are triangular symmetric fuzzy numbers. It is obvious that $(x, y)$ also is fuzzy numbers. Now, we want to find a point in one of the $K$ regions for a new machines such that the objective function of our considered problem in fuzzy case is minimized.

Hosseinzadeh et al. [26] consider the single facility case and present a linear programming for it.
For the fuzzy block norm case we have the following model.

$$
\begin{aligned}
& \min Z= \sum_{1 \leq j<k \leq n} v_{j k}\left\|\tilde{X}_{j}-\tilde{X}_{k}\right\|_{B} \\
&+\sum_{j=1}^{n} \sum_{i=1}^{m} w_{j i}\left\|\tilde{X}_{j}-\tilde{P}_{i}\right\|_{B} \\
& \text { S.t. } \tilde{a}_{i} \tilde{\leq} \tilde{x}_{j} \tilde{\leq} \tilde{b}_{i}, i=1, \cdots, K, j=1, \cdots, n \\
& \tilde{c}_{i} \tilde{\leq} \tilde{y}_{j} \tilde{\leq} \tilde{d}_{i}, i=1, \cdots, K, j=1, \cdots, n .
\end{aligned}
$$

Equivalently, according model (14) in Section 2 we have the following model for fuzzy numbers:

$$
\begin{aligned}
& \min Z=\sum_{1 \leq j<k \leq n g=1} \sum_{j k}^{r} v_{j k}\left(\tilde{\lambda}_{g j k}^{+}+\tilde{\lambda}_{g j k}^{-}\right)+\sum_{j=1}^{n} \sum_{i=1}^{m} \sum_{g=1}^{r} w_{j i}\left(\tilde{\beta}_{g j i}^{+}+\tilde{\beta}_{g j i}^{-}\right) \\
& \text {S.t. } \tilde{X}_{j}-\tilde{X}_{k}=\sum_{g=1}^{r}\left(\tilde{\lambda}_{g j k}^{+}-\tilde{\lambda}_{g j k}^{-}\right) b_{g}, \\
& \tilde{X}_{j}-\tilde{P}_{i}=\sum_{g=1}^{r}\left(\tilde{\beta}_{g j i}^{+}-\tilde{\beta}_{g j i}^{-}\right) b_{g} \\
& \tilde{x}_{j} \leq \tilde{b}_{i}+\tilde{M}\left(1-\gamma_{i j}\right), i=1, \cdots, K, j=1, \cdots, n \\
& \tilde{x}_{j} \geq \tilde{a}_{i}-\tilde{M}\left(1-\gamma_{i j}\right), i=1, \cdots, K, j=1, \cdots, n \\
& \tilde{y}_{j} \leq \tilde{d}_{i}+\tilde{M}\left(1-\gamma_{i j}\right), i=1, \cdots, K, j=1, \cdots, n \\
& \tilde{y}_{j} \geq \tilde{c}_{i}-\tilde{M}\left(1-\gamma_{i j}\right), i=1, \cdots, K, j=1, \cdots, n \\
& \sum_{i=1}^{K} \gamma_{i j}=1, j=1, \cdots, n ; \sum_{j=1}^{n} \gamma_{i j} \leq 1, i=1, \cdots, K, \gamma_{i j} \in\{0,1\}, \tilde{\lambda}_{g j k}^{+}, \tilde{\lambda}_{g j k}^{-}, \tilde{\beta}_{g j i}^{+}, \tilde{\beta}_{g j i}^{-} \geq 0 .
\end{aligned}
$$

The problem (19) can be converted to standard form as follows.

$$
\begin{aligned}
& \min Z=\sum_{1 \leq j<k \leq n g=1} \sum_{j k}^{r} v_{j k}\left(\tilde{\lambda}_{g j k}^{+}+\tilde{\lambda}_{g j k}^{-}\right)+\sum_{j=1}^{n} \sum_{i=1}^{m} \sum_{g=1}^{r} w_{j i}\left(\tilde{\beta}_{g j i}^{+}+\tilde{\beta}_{g j i}^{-}\right) \\
& \text {S.t. } \tilde{X}_{j}-\tilde{X}_{k}=\sum_{g=1}^{r}\left(\tilde{\lambda}_{g j k}^{+}-\tilde{\lambda}_{g j k}^{-}\right) b_{g}, \\
& \tilde{X}_{j}-\tilde{P}_{i}=\sum_{g=1}^{r}\left(\tilde{\beta}_{g j i}^{+}-\tilde{\beta}_{g j i}^{-}\right) b_{g} \\
& \tilde{x}_{j}+\tilde{u}_{i j}=\tilde{b}_{i}+\tilde{M}\left(1-\gamma_{i j}\right), i=1, \cdots, K, j=1, \cdots, n \\
& \tilde{x}_{j}-\tilde{u}_{i j}^{\prime}=\tilde{a}_{i}-\tilde{M}\left(1-\gamma_{i j}\right), i=1, \cdots, K, j=1, \cdots, n \\
& \tilde{y}_{j}+\tilde{v}_{i j}=\tilde{d}_{i}+\tilde{M}\left(1-\gamma_{i j}\right), i=1, \cdots, K, j=1, \cdots, n \\
& \tilde{y}_{j}-\tilde{v}_{i j}^{\prime}=\tilde{c}_{i}-\tilde{M}\left(1-\gamma_{i j}\right), i=1, \cdots, K, j=1, \cdots, n \\
& \sum_{i=1}^{K} \gamma_{i j}=1, j=1, \cdots, n ; \sum_{j=1}^{n} \gamma_{i j} \leq 1, i=1, \cdots, K, \gamma_{i j} \in\{0,1\}, \tilde{\lambda}_{g j k}^{+}, \tilde{\lambda}_{g j k}^{-}, \tilde{\beta}_{g j i}^{+}, \tilde{\beta}_{g j i}^{-} \geq 0 .
\end{aligned}
$$

In order to obtain the optimal solution of problem (20), we solve two problems in below: 


$$
\begin{aligned}
\min Z & =\sum_{1 \leq j<k \leq n g=1} \sum_{j k}^{r} v_{g j k}\left(\lambda_{g j k}^{+}\right)+\sum_{j=1}^{n} \sum_{i=1}^{m} \sum_{g=1}^{r} w_{j i}\left(\beta_{g j i}^{+}+\beta_{g j i}^{-}\right) \\
\text {S.t. } X_{j}-X_{k} & =\sum_{g=1}^{r}\left(\lambda_{g j k}^{+}-\lambda_{g j k}^{-} b_{g}\right), X_{j}-\operatorname{Core}(P)_{i}=\sum_{g=1}^{r}\left(\beta_{g j i}^{+}-\beta_{g j i}^{-}\right) b_{g} \\
x_{j}+u_{i j} & =\operatorname{Core}\left(b_{i}\right)+M\left(1-\gamma_{i j}\right), i=1, \cdots, K, j=1, \cdots, n \\
x_{j}-u_{i j}^{\prime} & =\operatorname{Core}\left(a_{i}\right)-M\left(1-\gamma_{i j}\right), i=1, \cdots, K, j=1, \cdots, n \\
y_{j}+v_{i j} & =\operatorname{Core}\left(d_{i}\right)+M\left(1-\gamma_{i j}\right), i=1, \cdots, K, j=1, \cdots, n \\
y_{j}-v_{i j}^{\prime} & =\operatorname{Core}\left(c_{i}\right)-M\left(1-\gamma_{i j}\right), i=1, \cdots, K, j=1, \cdots, n \\
\sum_{i=1}^{K} \gamma_{i j}=1, \quad j & =1, \cdots, n ; \sum_{j=1}^{n} \gamma_{i j} \leq 1, \quad i=1, \cdots, K, \gamma_{i j} \in\{0,1\}, \lambda_{g j k}^{+}, \lambda_{g j k}^{-}, \beta_{g j i}^{+}, \beta_{g j i}^{-} \geq 0, u_{i j}, u_{i j}^{\prime}, v_{i j}, v_{i j}^{\prime} \geq 0
\end{aligned}
$$

and

$$
\begin{aligned}
& \min Z=\sum_{1 \leq j<k \leq n g=1} \sum_{j k}^{r} v_{j j k}\left(\lambda_{g j k}^{+}+\lambda_{g j k}^{-}\right)+\sum_{j=1}^{n} \sum_{i=1}^{m} \sum_{g=1}^{r} w_{j i}\left(\beta_{g j i}^{+}+\beta_{g j i}^{-}\right) \\
& \text {S.t. } X_{j}+X_{k}+\sum_{g=1}^{r}\left(\lambda_{g j k}^{+}-\lambda_{g j k}^{-}\right) b_{g}=0, X_{j}+W\left(P_{i}\right)+\sum_{g=1}^{r}\left(\beta_{g j i}^{+}-\beta_{g j i}^{-}\right) b_{g}=0 \\
& \quad x_{j}+u_{i j}+M\left(1-\gamma_{i j}\right)=W\left(b_{i}\right), i=1, \cdots, K, j=1, \cdots, n \\
& \quad x_{j}-u_{i j}^{\prime}+M\left(1-\gamma_{i j}\right)=W\left(a_{i}\right), i=1, \cdots, K, j=1, \cdots, n \\
& y_{j}+v_{i j}+M\left(1-\gamma_{i j}\right)=W\left(d_{i}\right), i=1, \cdots, K, j=1, \cdots, n \\
& y_{j}-v_{i j}^{\prime}+M\left(1-\gamma_{i j}\right)=W\left(c_{i}\right), i=1, \cdots, K, j=1, \cdots, n \\
& \sum_{i=1}^{K} \gamma_{i j}=1, \quad j=1, \cdots, n ; \sum_{j=1}^{n} \gamma_{i j} \leq 1, \quad i=1, \cdots, K, \gamma_{i j} \in\{0,1\}, \lambda_{g j k}^{+}, \lambda_{g j k}^{-}, \beta_{g j i}^{+}, \beta_{g j i}^{-} \geq 0, u_{i j}, u_{i j}^{\prime}, v_{i j}, v_{i j}^{\prime} \geq 0
\end{aligned}
$$

Note that Theorem 4.4 is hold when the optimal values of the variables $\gamma_{i j}$ in models (21) and (22) are the same.

In the case norm is rectelinear we have the following nonlinear model:

$$
\begin{aligned}
& \min Z=\sum_{1 \leq j<k \leq n} v_{j k}\left|\tilde{x}_{j}-\tilde{x}_{k}\right|+\sum_{j=1}^{n} \sum_{i=1}^{m} w_{j i}\left|\tilde{x}_{j}-p \ddot{x}_{i}\right|+\sum_{1 \leq j<k \leq n} v_{j k}\left|\tilde{y}_{j}-\tilde{y}_{k}\right|+\sum_{j=1}^{n} \sum_{i=1}^{m} w_{j i}\left|\tilde{y}_{j}-p \ddot{y}_{i}\right| \\
& \text { S.t. } \quad \tilde{a}_{i} \tilde{\leq} \tilde{x}_{j} \tilde{\leq} \tilde{b}_{i}, i=1, \cdots, K, j=1, \cdots, n, \\
& \quad \tilde{c}_{i} \tilde{y}_{j} \tilde{y}_{j} \tilde{d}_{i}, i=1, \cdots, K, j=1, \cdots, n .
\end{aligned}
$$

Equivalently, according model (12) in Section 2 we have the below model for fuzzy numbers:

$$
\begin{aligned}
& \min Z=\sum_{1 \leq j<k \leq n} v_{j k}\left(\tilde{p}_{j k}+\tilde{q}_{j k}\right)+\sum_{j=1}^{n} \sum_{i=1}^{m} w_{j i}\left(\tilde{r}_{j i}+\tilde{s}_{j i}\right)+\sum_{1 \leq j<k \leq n} v_{j k}\left(\tilde{p}_{j k}^{\prime}+\tilde{q}_{j k}^{\prime}\right)+\sum_{j=1}^{n} \sum_{i=1}^{m} w_{j i}\left(\tilde{r}_{j i}^{\prime}+\tilde{s}_{j i}^{\prime}\right) \\
& \text { S.t. } \tilde{x}_{j}-\tilde{x}_{k}-\tilde{p}_{j k}+\tilde{q}_{j k}=0, \tilde{y}_{j}-\tilde{y}_{k}-\tilde{p}_{j k}^{\prime}+\tilde{q}_{j k}^{\prime}=0 \\
& \tilde{x}_{j}-\tilde{r}_{j i}+\tilde{s}_{j i}=p \ddot{x}_{i}, \tilde{y}_{j}-\tilde{r}_{j i}^{\prime}+\tilde{s}_{j i}^{\prime}=p \ddot{y}_{i} \\
& \tilde{x}_{j} \tilde{\leq} \tilde{b}_{i}+\tilde{M}\left(1-\gamma_{i j}\right), i=1, \cdots, K, j=1, \cdots, n \\
& \tilde{x}_{j} \geq \tilde{a}_{i}-\tilde{M}\left(1-\gamma_{i j}\right), i=1, \cdots, K, j=1, \cdots, n \\
& \tilde{y}_{j} \tilde{\leq} \tilde{d}_{i}+\tilde{M}\left(1-\gamma_{i j}\right), i=1, \cdots, K, j=1, \cdots, n \\
& \tilde{y}_{j} \tilde{\geq} \tilde{c}_{i}-\tilde{M}\left(1-\gamma_{i j}\right), i=1, \cdots, K, j=1, \cdots, n \\
& \sum_{i=1}^{K} \gamma_{i j}=1, j=1, \cdots, n ; \sum_{j=1}^{n} \gamma_{i j} \leq 1, \quad i=1, \cdots, K, \gamma_{i j} \in\{0,1\} n, \tilde{p}_{j k}, \tilde{q}_{j k}, \tilde{p}_{i j}^{\prime}, \tilde{q}_{i j}^{\prime}, \tilde{r}_{j i}, \tilde{s}_{j i}, \tilde{r}_{i j}^{\prime}, \tilde{s}_{i j}^{\prime} \geq 0 .
\end{aligned}
$$


The problem (24) can be converted to standard form as:

$$
\begin{aligned}
& \min Z=\sum_{1 \leq j<k \leq n} v_{j k}\left(\tilde{p}_{j k}+\tilde{q}_{j k}\right)+\sum_{j=1}^{n} \sum_{i=1}^{m} w_{j i}\left(\tilde{r}_{j i}+\tilde{s}_{j i}\right)+\sum_{1 \leq j<k \leq n} v_{j k}\left(\tilde{p}_{j k}^{\prime}+\tilde{q}_{j k}^{\prime}\right)+\sum_{j=1}^{n} \sum_{i=1}^{m} w_{j i}\left(\tilde{r}_{j i}^{\prime}+\tilde{s}_{j i}^{\prime}\right) \\
& \text { S.t. } \tilde{x}_{j}-\tilde{x}_{k}-\tilde{p}_{j k}+\tilde{q}_{j k}=0, \tilde{y}_{j}-\tilde{y}_{k}-\tilde{p}_{j k}^{\prime}+\tilde{q}_{j k}^{\prime}=0 \\
& \tilde{x}_{j}-\tilde{r}_{j i}+\tilde{s}_{j i}=p \ddot{x}_{i}, \tilde{y}_{j}-\tilde{r}_{j i}^{\prime}+\tilde{s}_{j i}^{\prime}=p \ddot{y}_{i} \\
& \tilde{x}_{j}+\tilde{u}_{i j}=\tilde{b}_{i}+\tilde{M}\left(1-\gamma_{i j}\right), i=1, \cdots, K, j=1, \cdots, n \\
& \tilde{x}_{j}-\tilde{u}_{i j}^{\prime}=\tilde{a}_{i}-\tilde{M}\left(1-\gamma_{i j}\right), i=1, \cdots, K, j=1, \cdots, n \\
& \tilde{x}_{j}+\tilde{v}_{i j}=\tilde{d}_{i}+\tilde{M}\left(1-\gamma_{i j}\right), i=1, \cdots, K, j=1, \cdots, n \\
& \tilde{x}_{j}-\tilde{v}_{i j}^{\prime}=\tilde{c}_{i}-\tilde{M}\left(1-\gamma_{i j}\right), i=1, \cdots, K, j=1, \cdots, n \\
& \sum_{i=1}^{K} \gamma_{i j}=1, \quad j=1, \cdots, n ; \sum_{j=1}^{n} \gamma_{i j} \leq 1, \quad i=1, \cdots, K, \gamma_{i j} \in\{0,1\} \\
& \tilde{p}_{i j}, \tilde{q}_{i j}, \tilde{p}_{i j}^{\prime}, \tilde{q}_{i j}^{\prime} \geq 0 ; \tilde{r}_{i j}, \tilde{s}_{i j}, \tilde{r}_{i j}^{\prime}, \tilde{s}_{i j}^{\prime} \geq 0 ; \tilde{u}_{i j}, \tilde{u}_{i j}^{\prime}, \tilde{v}_{i j}, \tilde{r}_{i j}^{\prime} \geq 0 .
\end{aligned}
$$

In order to obtain the optimal solution of problem (25), we solve two problems in below:

$$
\begin{aligned}
& \min Z=\sum_{1 \leq j<k \leq n} v_{j k}\left(p_{j k}+q_{j k}\right)+\sum_{j=1}^{n} \sum_{i=1}^{m} w_{j i}\left(r_{j i}+s_{j i}\right)+\sum_{1 \leq j<k \leq n} v_{j k}\left(p_{j k}^{\prime}+q_{j k}^{\prime}\right)+\sum_{j=1}^{n} \sum_{i=1}^{m} w_{j i}\left(r_{j i}^{\prime}+s_{j i}^{\prime}\right) \\
& \text { S.t. } x_{j}-x_{k}-p_{j k}+q_{j k}=0, y_{j}-y_{k}-p_{j k}^{\prime}+q_{j k}^{\prime}=0 \\
& x_{j}-r_{j i}+s_{j i}=\operatorname{core}\left(p x_{i}\right), y_{j}-r_{j i}^{\prime}+s_{j i}^{\prime}=\operatorname{core}\left(p y_{i}\right) \\
& x_{j}+u_{i j}=\operatorname{core}\left(b_{i}\right)+M\left(1-\gamma_{i j}\right), i=1, \cdots, K, j=1, \cdots, n \\
& x_{j}-u_{i j}^{\prime}=\operatorname{core}\left(a_{i}\right)-M\left(1-\gamma_{i j}\right), i=1, \cdots, K, j=1, \cdots, n \\
& y_{j}+v_{i j}=\operatorname{core}\left(d_{i}\right)+M\left(1-\gamma_{i j}\right), i=1, \cdots, K, j=1, \cdots, n \\
& y_{j}-v_{i j}^{\prime}=\operatorname{core}\left(c_{i}\right)-M\left(1-\gamma_{i j}\right), i=1, \cdots, K, j=1, \cdots, n \\
& \sum_{i=1}^{K} \gamma_{i j}=1, \quad j=1, \cdots, n ; \sum_{j=1}^{n} \gamma_{i j} \leq 1, \quad i=1, \cdots, K, \gamma_{i j} \in\{0,1\} \\
& p_{i j}, q_{i j}, p_{i j}^{\prime}, q_{i j}^{\prime} \geq 0 ; r_{i j}, s_{i j}, r_{i j}^{\prime}, s_{i j}^{\prime} \geq 0 ; u_{i j}, u_{i j}^{\prime}, v_{i j}, v_{i j}^{\prime} \geq 0 .
\end{aligned}
$$

and

$$
\begin{aligned}
& \min Z=\sum_{1 \leq j<k \leq n} v_{j k}\left(p_{j k}+q_{j k}\right)+\sum_{j=1}^{n} \sum_{i=1}^{m} w_{j i}\left(r_{j i}+s_{j i}\right)+\sum_{1 \leq j<k \leq n} v_{j k}\left(p_{j k}^{\prime}+q_{j k}^{\prime}\right)+\sum_{j=1}^{n} \sum_{i=1}^{m} w_{j i}\left(r_{j i}^{\prime}+s_{j i}^{\prime}\right) \\
& \text { S.t. } x_{j}+x_{k}+p_{j k}+q_{j k}=0, y_{j}+y_{k}+p_{j k}^{\prime}+q_{j k}^{\prime}=0 \\
& x_{j}+r_{j i}+s_{j i}=W\left(p x_{i}\right), y_{j}+r_{j i}^{\prime}+s_{j i}^{\prime}=W\left(p y_{i}\right) \\
& x_{j}+u_{i j}+M \gamma_{i j}=W\left(b_{i}\right)+M, i=1, \cdots, K, j=1, \cdots, n \\
& x_{j}+u_{i j}^{\prime}+M \gamma_{i j}=W\left(a_{i}\right)-M, i=1, \cdots, K, j=1, \cdots, n \\
& y_{j}+v_{i j}+M \gamma_{i j}=W\left(d_{i}\right)+M, i=1, \cdots, K, j=1, \cdots, n \\
& y_{j}+v_{i j}^{\prime}+M \gamma_{i j}=W\left(c_{i}\right)-M, i=1, \cdots, K, j=1, \cdots, n \\
& \sum_{i=1}^{K} \gamma_{i j}=1, \quad j=1, \cdots, n ; \sum_{j=1}^{n} \gamma_{i j} \leq 1, \quad i=1, \cdots, K, \gamma_{i j} \in\{0,1\} \\
& p_{j k}, q_{j k}, p_{j k}^{\prime}, q_{j k}^{\prime} \geq 0 ; r_{j i}, s_{j i}, r_{j i}^{\prime}, s_{j i}^{\prime} \geq 0 ; u_{i j}, u_{i j}^{\prime}, v_{i j}, v_{i j}^{\prime} \geq 0 .
\end{aligned}
$$

Again the theorem 4.4 is hold when the optimal values of the variables $\gamma_{i j}$ in models (26) and (27) are the 
same.

\section{Summary and Conclusion}

In this paper we presented a linear model for finding optimal locations for multiple facilities with respect to the other facilities and then use it for fuzzy data. Here we use block norm and rectilinear norm as a special case, for finding the optimal locations. Also in order to avoid congestion, we suppose that an eligible site must be as interval. Finally, in order to locate new facilities in convex sets, we suggest a model by which the weighted distance between new facilities and all the other existing facilities and the new ones is minimized.

\section{REFERENCES}

[1] R. E. Bellman and L. A. Zadeh, "Decision Making in a Fuzzy Environment," Management Science, Vol. 17, No. 4, 1970, pp. 141-164. doi:10.1287/mnsc.17.4.B141

[2] Z. Drezner and H. Hamacher, "Facility Location: Applications and Theory," Springer-Verlag, Berlin, 2002.

[3] A. Sarkar, R. Batta and R. Nagi, "Placing a Finite Size Facility with a Center Objective on a Rectangular Plane with Barriers," European Journal of Operational Research, Vol. 179, No. 3, 2007, pp. 1160-1176. doi:10.1016/j.ejor.2005.08.029

[4] R. C. Larson and G. Sadiq, "Facility Locations with the Manhattan Metric in the Presence of Barriers to Travel," Operations Research, Vol. 31, No. 4, 1983, pp. 652-669. doi:10.1287/opre.31.4.652

[5] J. Muñoz-Pérez and J. J. Saameño-Rodríguez, "Location of an Undesirable Facility in a Polygonal Region with Forbidden Zones," European Journal of Operational Research, Vol. 114, No. 2, 1999, pp. 372-379. doi:10.1016/S0377-2217(98)00138-6

[6] U. Bhattacharya, J. R. Rao and R. N. Tiwari, "Fuzzy Multi-Criteria Facility Location Problem," Fuzzy Sets and Systems, Vol. 51, No. 3, 1992, pp. 277-287. doi:10.1016/0165-0114(92)90018-Y

[7] U. Bhattacharya, J. R. Rao and R. N. Tiwari, "Bi-Criteria Multi Facility Location Problem in Fuzzy Environment," Fuzzy Sets and Systems, Vol. 56, No. 2, 1993, pp. 145153. doi:10.1016/0165-0114(93)90139-9

[8] S. M. A. Nayeem and M. Pal, "The p-Center Problem on Fuzzy Networks and Reduction of Cost," Iranian Journal of Fuzzy Systems, Vol. 5, No. 1, 2008, pp. 1-26.

[9] R. Francis Jr., L. F. McGinnis and J. A. White, "Facility Layout and Location: An Analytical Approach," Prentice Hall, Upper Saddle River, 1992.

[10] G. Y. Handler and P. B. Mirchandani, "Location on Networks: Theory and Algorithms," MIT Press, Cambridge, 1979.

[11] R. F. Love, J. G. Morris and G. O. Wesolowsky, "Facilities Location: Models and Methods," North Holland Pub- lishing Company, New York, 1988.

[12] P. B. Mirchandani and R. Francis, "Discrete Location Theory," John Wiley \& Sons, Hoboken, 1990.

[13] J. E. Ward and R. E. Wendell, "A New Norm for Measuring Distance Which Yields Linear Location Problems," Operations Research, Vol. 28, No. 3, 1980, pp. 836-844. doi:10.1287/opre.28.3.836

[14] J. E. Ward and R. E. Wendell, "Using Block Norms for Location Modeling," Operations Research, Vol. 33, No. 5, 1985, pp. 1074-1090. doi:10.1287/opre.33.5.1074

[15] H. Tanaka, T. Okuda and K. Asai, "On Fuzzy Mathematical Programming," Journal of Cybernetics, Vol. 3, No. 4, 1973, pp. 37-46. doi:10.1080/01969727308545912

[16] H. J. Zimmermann, "Fuzzy Mathematical Programming," Computers \& Operations Research Journal, Vol. 10, No. 4, 1993, pp. 291-298. doi:10.1016/0305-0548(83)90004-7

[17] C. V. Negoita, "Fuzziness in Management," OPSA/TIMS, Miami, 1970.

[18] H. Tanaka and K. Asai, "Fuzzy Linear Programming Problems with Fuzzy Numbers," Fuzzy Sets and Systems, Vol. 13, No. 1, 1984, pp. 1-10. doi:10.1016/0165-0114(84)90022-8

[19] H. R. Maleki, M. Tata and M. Mashinchi, "Linear Programming with Fuzzy Variables," Fuzzy Sets and Systems, Vol. 109, No. 1, 2000, pp. 21-33. doi:10.1016/S0165-0114(98)00066-9

[20] S. C. Fang and C. F. Hu, "Linear Programming with Fuzzy Coefficients in Constraints," Computers \& Mathematics with Applications, Vol. 37, No. 10, 1999, pp. 63-76. doi:10.1016/S0898-1221(99)00126-1

[21] R. Fuller and H. J. Zimmermann, "Fuzzy Reasoning for Solving Fuzzy Mathematical Programming Problems," Fuzzy Sets and Systems, Vol. 60, No. 2, 1993, pp. 121133. doi:10.1016/0165-0114(93)90341-E

[22] R. N. Gasimov and K. Yenilmez, "Solving Fuzzy Linear Programming Problems with Linear Membership Function," Turkish Journal of Mathematics, Vol. 26, No. 4, 2002, pp. 375-396.

[23] C. Garsia-Aguado and J. L. Verdegay, "On the Sensitivity of Membership Functions for Fuzzy Linear Programming Problems," Fuzzy Sets and Systems, Vol. 56, No. 1, 1993, pp. 47-49. doi:10.1016/0165-0114(93)90184-J

[24] H. R. Maleki, "Ranking Functions and Their Applications to Fuzzy Linear Programming," Far East Journal of Mathematical Sciences, Vol. 4, No. 3, 2002, pp. 283-301.

[25] H. Mishmast Nehi, H. R. Maleki and M. Mashinchi, "Solving Fuzzy Number Linear Programming Problem by Lexicographic Ranking Function," Italian Journal of Pure and Applied Mathematics, Vol. 15, 2004, pp. 9-20.

[26] F. Hosseinzadeh Lotfi, G. R. Jahanshahloo, F. Rezai Balf and H. Zhiani Rezai, "Finding the Minimize Summation for Location of Facility in a Convex Set with Fuzzy Data," Applied Mathematical Sciences, Vol. 16, No. 1, 2007, pp. 749-759. 